\title{
Investigation of Cobalt Oxidation States in PtCo Nanoparticles Using STEM-EELS
}

\author{
James O. Sode ${ }^{1}$, Rebecca J. Nicholls ${ }^{2}$, Peter D. Nellist ${ }^{1}$, Trung Tran $^{2}$ and Dogan Ozkaya ${ }^{2}$ \\ 1. University of Oxford, Department of Materials, Oxford, UK. \\ 2. Johnson Matthey Technology Centre, Sonning Common, Reading, UK.
}

For efficient conversion in hydrogen fuel cells, the interaction of oxygen with the catalyst is important. The interfacial chemistry of the nanoparticles (NPs) at the nanometre scale is crucial to truly understand the oxygen reduction reaction (ORR), the limiting step of catalytic activity in Pt catalysts [1]. In order to achieve this, the characterisation techniques of aberration-corrected Scanning Transmission Electron Microscopy (STEM) and Electron Energy Loss Spectroscopy (EELS) are employed. Using spectrum imaging, a combination of elemental maps and spectra can be used to investigate the bonding and location of the oxygen atoms towards the surface of catalyst nanoparticles.

In STEM-EELS spectra, sometimes the oxygen $K$-edge signal is unquantifiable due to weak signal-tonoise ratio. When this is the case, the white lines of the metals in which they are bonded to, in this case cobalt $L_{2,3}$-edges, can be used to elucidate the location of oxygen in the NPs. The platinum $M_{4,5}$-edge is broad and not useful. The variation in the cobalt $L_{2,3}$ ratios are analysed at a higher spectral dispersion than data with the oxygen $K$-edge. However, as there is not a one-to-one correspondence between the ratio and oxidation state as determined from measured cobalt standards and literature, ambiguity arises when trying to determine oxidation states solely with the $L_{2,3}$ ratios. Consequently, the shapes and energies of the cobalt $L_{2,3}$-edges, together with oxygen $K$-edge (when resolvable) is used to estimate the oxidation states.

This work reports on as-received $\mathrm{Pt}_{3} \mathrm{Co}$ and $\mathrm{PtCo}_{3}$ (leached and unleached) NPs. These were chosen to examine the effect of alloying on the oxygen binding sites and thus, catalytic activity, stemming from previously published literature [2]. The cobalt $L_{2,3}$ intensities in the aberration-corrected STEM images were integrated over different pixel areas, represent as cores and shells, and their ratios calculated. The particles were measured to get a sense of variation with the size of the NPs. Finally, the multiplet splittings of the cobalt $L_{2,3}$-edges, as well as the shape of the oxygen $K$-edge were used to determine trends in the oxidation of the $\mathrm{Pt}_{3} \mathrm{Co}$ and $\mathrm{PtCo}_{3} \mathrm{NPs}$.

All images and spectra were recorded between the Oxford Materials JEOL ARM200CF and the Harwell Diamond JEOL ARM200CF. The microscopes were operated at 200kV acceleration voltage. Simultaneous structural and compositional data were recorded using the ADF detector and $5 \mathrm{~mm}$ aperture Gatan EELS GIF Quantum system in the DUAL-EELS setup. The ADF detector inner and outer angles were $\sim 108$ and $553 \mathrm{mrad}$ respectively, while the EELS convergence and collection angles were $\sim 23$ and $56 \mathrm{mrad}$ respectively. The EELS signal was recorded using both $0.25 \mathrm{eV}$ and $0.025 \mathrm{eV}$ dispersion for cobalt $L_{2,3}$-edge data with and without the oxygen $K$-edge. The core-loss spectra were recorded with a pixel dwell time between $0.05-0.02 \mathrm{~s}$, whereas the low-loss spectra were recorded with a pixel dwell time between 10$40 \mu \mathrm{s}$.

Major findings were that while small $(\leq 5 \mathrm{~nm})$ particles had more variation and higher area of oxidation, the oxidation of large particles $(\geq 8 \mathrm{~nm})$ was mainly restricted to the edge/shell with the core being very ordered and metallic. One such particle is shown in figure 1 illustrating this phenomenon. Figure 2 corroborates this finding as noticeable from the cobalt $L_{2,3}$-edge and oxygen $K$-edge EELS spectra. It was 
also observed that the small particles on average had higher oxidation states than the large nanoparticles. Similar to the effect of size, $\mathrm{PtCo}_{3}$ were found to have higher areas and states of oxidation than $\mathrm{Pt}_{3} \mathrm{Co}$ nanoparticles. Lastly, leached nanoparticles varied in oxidation a lot more than unleached nanoparticles.

\section{References:}

[1] V Stamenkovic et al., Angewandte Chemie 118 (2006), p. 2963.

[2] C Wang et al., Physical Chemistry 12 (2010), p. 6933.

(a)

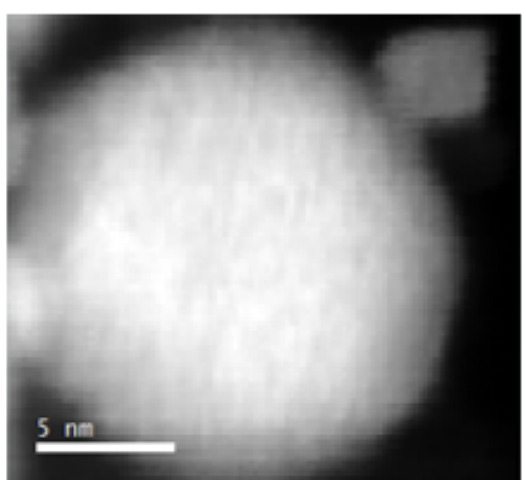

(b)

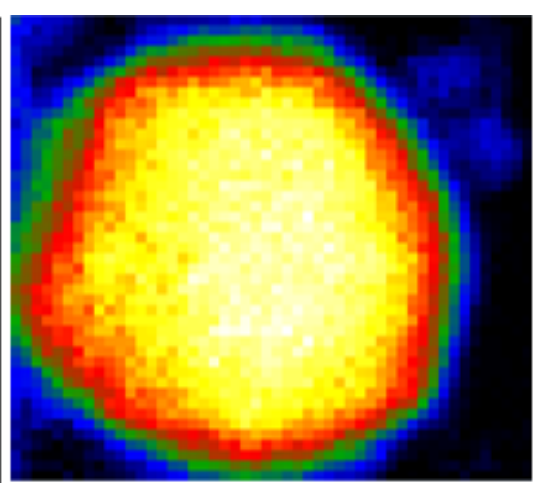

(c)

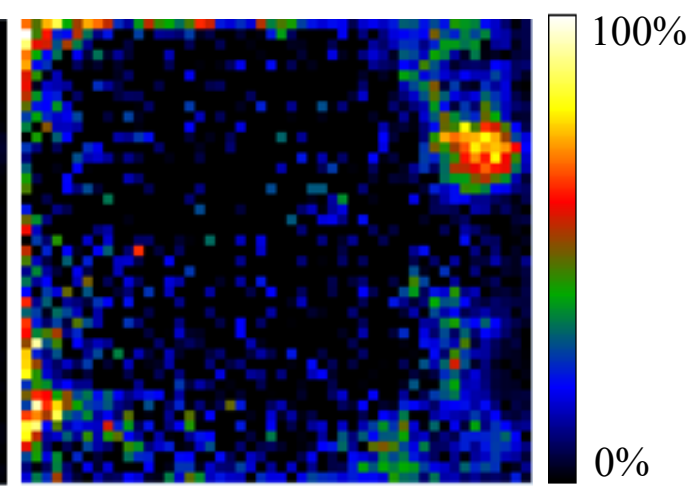

Figure 1. HAADF image of a $\mathrm{PtCo}_{3}$ unleached nanoparticle (a), EELS cobalt $L_{2,3}$-edge false colour map (778.1 -809.6 eV) (b) and EELS oxygen $K$-edge false colour map (530.29-580.89 eV) (c).
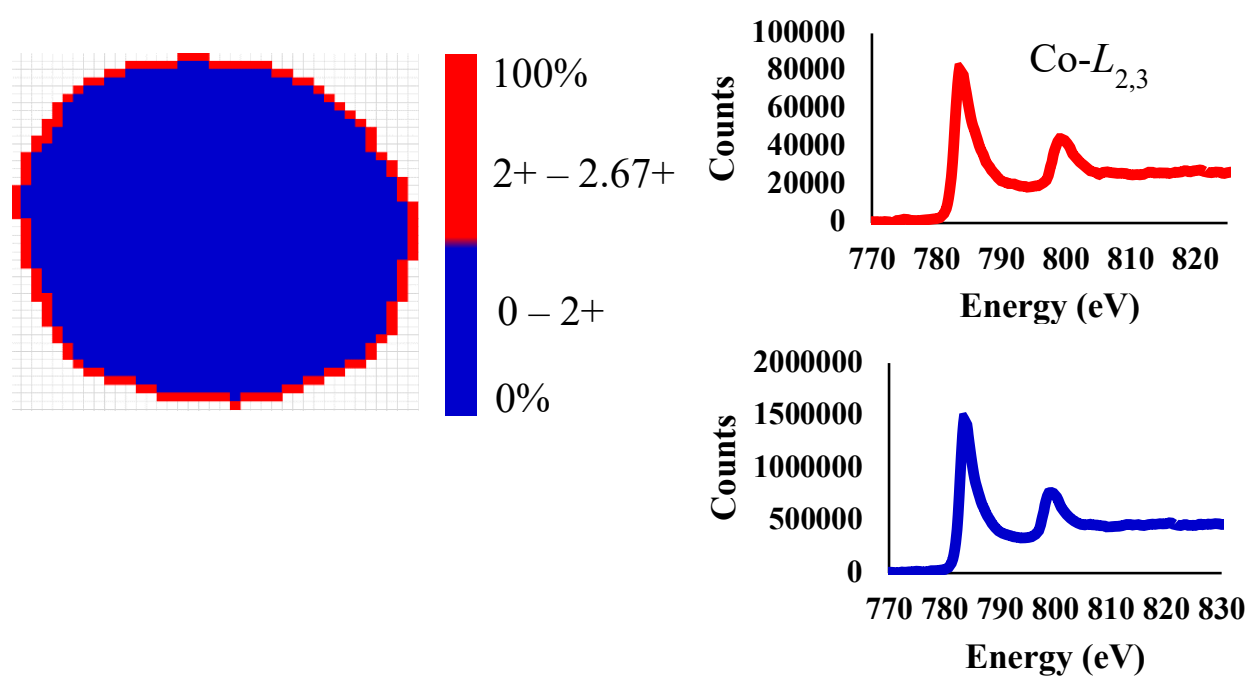
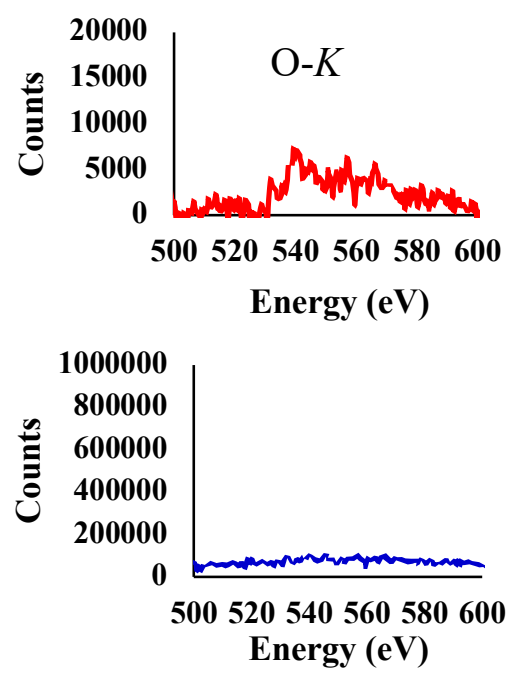

Figure 2. Core-shell binary false colour image of the nanoparticle in figure 1 together with the cobalt $L_{2,3}$-edge and oxygen $K$-edge EELS spectra from the core and shell regions. 